\title{
Flavonoids and the Structure-Antioxidant Activity Relationship
}

Daniela Russo*

Department of Science, University of Basilicata, Potenza, Italy

*Corresponding author: Daniela Russo, Department of Science, Basilicata University, Potenza, Italy, E-mail: daniela.russo@unibas.it

Received date: February 16, 2018; Accepted date: February 22, 2018; Published date: February 23, 2018

Copyright: (c) 2018 Russo D. This is an open-access article distributed under the terms of the Creative Commons Attribution License, which permits unrestricted use, distribution, and reproduction in any medium, provided the original author and source are credited.

\section{Editorial}

For thousands of years, plants have been well documented for their medicinal uses and nowadays, about $80 \%$ of world population is dependent on traditional medicines [1]. The knowledge associated with traditional medicine has promoted further investigations of medicinal plants as potential medicines and has led to the isolation of many natural products. Herbal medicine materials consist in juices, gums, essential oils and any other natural substances containing as bioactive components, underground and aerial plant parts or other plant materials, in the crude state or as plant preparations. Among all natural bioactive products, flavonoids represent a group of phenolic compounds widely distributed in the plant kingdom and they have a positive effect on human health. In plants, flavonoids are synthetized via phenylpropanoid biosynthetic pathway and their structural feature is a 2-phenyl-benzo- $\gamma$-pyrane nucleus including two aromatic rings (A and $\mathrm{B}$ rings) and a pyran heterocyclic ring ( $\mathrm{C}$ ring). The A-ring originates from the cyclization of 3 molecules of malonyl CoA and it is linked to B-ring, derived from phenylalanine, by a three-carbon bridge (C-ring).

The great variability of the flavonoids is due to the differences in the ring structure of the aglycone and in its state of oxidation/reduction, in the hydroxylation state of the aglycone and in the positions of the hydroxyl groups and in the derivatisation of the hydroxyl groups with methyl groups, carbohydrates, or isoprenoids. The main substituents are hydroxyl, methoxyl, or glycosyl, which can be further, substituted forming chemical structures very complex. Classification of flavonoids into subclasses is based on the functional groups in the $\mathrm{C}$ ring and they include anthocyanidins, flavanols, flavones, flavonols, flavanones, and isoflavonoids.

The antioxidant properties of flavonoids are important in determining their role as protective agents against free radicals. They are able to scavenge a wide range of reactive species, including hydroxyl radicals and superoxide radical and they can also inhibit biomolecular damage by peroxynitrite. They are also known to exhibit antibacterial effects, enzyme inhibition, antimicrobial activity, antimutagenic and anti-inflammatory activities in bacteria and mammalian, respectively [2]. Proanthocyanidin-rich extracts from grape seeds also display anticataract activity in rats [3]. Due to their antioxidant activity, flavonoids have been reported to have positive effects on cancer, cardiovascular disease, immune disorders, microbial infections, neurogenerative disease and viral infections [4-7]. They can participate in protection against the harmful action of ROS (Radical Oxygen Species); further they have multiple applications in food, cosmetic and pharmaceutical industries.

The structure of flavonoids is a key determinant of their antioxidant activities. The antioxidant activity of flavonoids increases with increasing degree of hydroxylation. However, substitution of the hydroxyl groups at the 3- and 5-position with methoxyl groups reduces the activity. Some structural features and nature of substitutions on rings $\mathrm{B}$ and $\mathrm{C}$ determine the antioxidant activity of flavonoids. The degree of hydroxylation and the positions of the - $\mathrm{OH}$ groups in the $\mathrm{B}$ ring, as an ortho-dihydroxyl structure of ring B (catechol group) leads to higher activity, or acts as the preferred binding site for trace metals [8]. The presence of hydroxyl groups at the $3^{\prime}-$-, 4 '-, and 5'-positions of ring $\mathrm{B}$ (a pyrogallol group) was found to enhance the antioxidant activity. A double bond between C-2 and C-3, conjugated with the 4oxo group in ring $\mathrm{C}$ improves the radical scavenging capacity of flavonoids [8] and the double bond between C-2 and C-3, combined with a $3-\mathrm{OH}$ (ring $\mathrm{C}$ ), also increases the active radical scavenging capacity of flavonoids. The substitution of hydroxyl groups in ring B by methoxyl groups alters the redox potential, which affects the radical scavenging capacity of flavonoids $[8,9]$. It was demonstrated that in flavonoids the O-glycosylation decreases their antioxidant potential in in vitro assays, whereas the C-glycosylation in most cases increases their antioxidant potential $[10,11]$. Blocking the hydroxy group at the C-3 position or removing the $3-\mathrm{OH}$ group decreases antioxidative properties of flavonoids. Further studies are focused on the discovery of new flavonoids from plant material and their structure-activity relationship.

Due to the traditional medicine, plants still represent a large source of natural compounds, new chemical entities, for the development of novel drugs [12]. Further studies are focused on the discovery of new bioactive compounds as flavonoids [13] from plant material and their structure-activity relationship. The knowledge of the structure-activity relationship is a powerful concept in drug discovery; it is involved in the selection and optimization of ideal drug candidates [14].

\section{References}

1. Oyebode O, Kandala NB, Chilton PJ, Lilford RJ (2016) Use of traditional medicine in middle-income countries: a WHO-SAGE study. Health Policy and Planning 31: 984-991.

2. Kaur S, Grover IS, Kumar S (1997) Antimutagenic potential of ellagic acid isolated from Terminalia arjuna. Indian J Exp Biol. 35: 478-482.

3. Yamakoshi J, Saito M, Kataoka S, Tokutake S (2002) Procyanidin-rich extract from grape seeds prevents cataract formation in hereditary cataractous (ICR/f) rats. J Agric Food Chem 50: 4983-4988.

4. Fidelis QC, Faraone I, Russo D, Aragão Catunda-Jr FE, Vignola L, et al. (2018) Chemical and Biological insights of Ouratea hexasperma (A. St.Hil.) Baill.: a source of bioactive compounds with multifunctional properties. Natural product research 1-4.

5. Akhavan M, Jahangiri S, Shafaghat A (2015) Studies on the antioxidant and antimicrobial activity and flavonoid derivatives from the fruit of Trigonosciadium brachytaenium (Boiss.) Alava. Industrial crops and Products. 63: 114-118.

6. Costa SL, Silva VDA, dos Santos Souza C, Santos CC, Paris I, et al. (2016). Impact of plant-derived flavonoids on neurodegenerative diseases. Neurotoxicity Res 30: 41-52. 
Citation: Russo D (2018) Flavonoids and the Structure-Antioxidant Activity Relationship. J Pharmacogn Nat Prod 4: e109. doi: 10.4172/2472-0992.1000e109

Page 2 of 2

7. Kumar S, Pandey AK (2013) Chemistry and biological activities of flavonoids: an overview. The Scientific World Journal, 2013.

8. Pietta PG (2000) Flavonoids as antioxidants. J Nat Prod. 63: 1035-1042.

9. Seeram NP, Nair MG (2002) Inhibition of lipid peroxidation and structureactivity-related studies of the dietary constituents anthocyanins, anthocyanidins, and catechins. J Agric Food Chem 50: 5308-5312.

10. Xiao J, Chen T, Cao H (2014) Flavonoid glycosylation and biological benefits. Biotechnol Adv.

11. Xiao J (2017) Dietary flavonoid aglycones and their glycosides: which show better biological significance?. Critical Reviews in Food Science and Nutrition 57: 1874-1905.
12. Dar RA, Shahnawaz M, Rasool S, Qazi PH (2017) Natural product medicines: A literature update. The Journal of Phytopharmacology 6: 340-342.

13. Xu HX, Wan M, Dong H, But PP, Foo LY, et al. (2000) Inhibitory activity of flavonoids and tannins against HIV-1 protease. Biol Pharm Bull 23: 1072-1076.

14. Mezrag A, Malafronte N, Bouheroum M, Travaglino C, Russo D, et al. (2017) Phytochemical and antioxidant activity studies on Ononis angustissima L. aerial parts: isolation of two new flavonoids. Nat Prod Res. 31: $507-514$ 